\section{Transient left septal fascicular block in the scenario of ST-segment elevation myocardial infarction}

\author{
Shandong Yu (D), Hui Chen (D), Hongwei Li (D) \\ Department of Cardiology, Cardiovascular Center, Beijing Friendship \\ Hospital, Capital Medical University; Beijing-China
}

\section{Introduction}

Although the conduction abnormalities of the left anterior and posterior fascicles, left anterior fascicular block (LAFB), and left posterior fascicular block (LPFB) are commonly diagnosed and well-known to physicians, the abnormalities of the left septal fascicle (LSF) and left septal fascicular block (LSFB), are easily overlooked because most physicians are not aware of the existence of the LSF. LSFB exclusively affects precordial leads without modifying the ECG in the frontal plane, causing prominent anterior QRS forces. LSF is irrigated exclusively by the left anterior descending (LAD) coronary artery, and therefore, LAD occlusion can result in LSFB. We report a case with transient LSFB in the scenario of ST-segment elevation myocardial infarction (STEMI) because of acute occlusion of LAD.

\section{Case Report}

A 69-year-old female patient with a history of hypertension, diabetes, and hyperglycemia presented to the emergency department with sudden chest pain for 3 hours. Her mother and brother had a history of coronary heart disease. Furthermore, her brother had received percutaneous coronary intervention (PCI) for treating his underlying heart condition. On admission, her physical examination was unremarkable. Her blood pressure was 112/68 mm Hg and heart rate was 79 beats/min. A baseline ECG was performed and is shown in Figure 1. Her cardiac troponin I level was $0.055 \mathrm{ng} / \mathrm{mL}$ (normal, $<0.02 \mathrm{ng} / \mathrm{mL}$ ). The patient was sent to the catheterization laboratory. On the basis of the ECG, we aimed to identify the culprit lesion, and determine why the precordial R/S transition from lead V1 to V2 was so early. The baseline ECG (Fig. 1) showed the precordial R wave "in crescendo" from V1 through V3 and decreasing from V4 to V6; and ST-segment elevation from V2 to V4. The " $R$ " wave jump occurred from V1 to V2. The ECG revealed STEMI caused by total occlusion of the LAD coronary artery. The coronary angiography showed total occlusion of LAD (Fig. 2a). After a successful $\mathrm{PCl}$ was performed (Fig. 2b), ECG demonstrated an "R" wave jump from V2 to V3 (Fig. 3). An ECG recorded the next day showed ST-segment resolution and T wave inversion of V2 to V4 (Fig. 4).

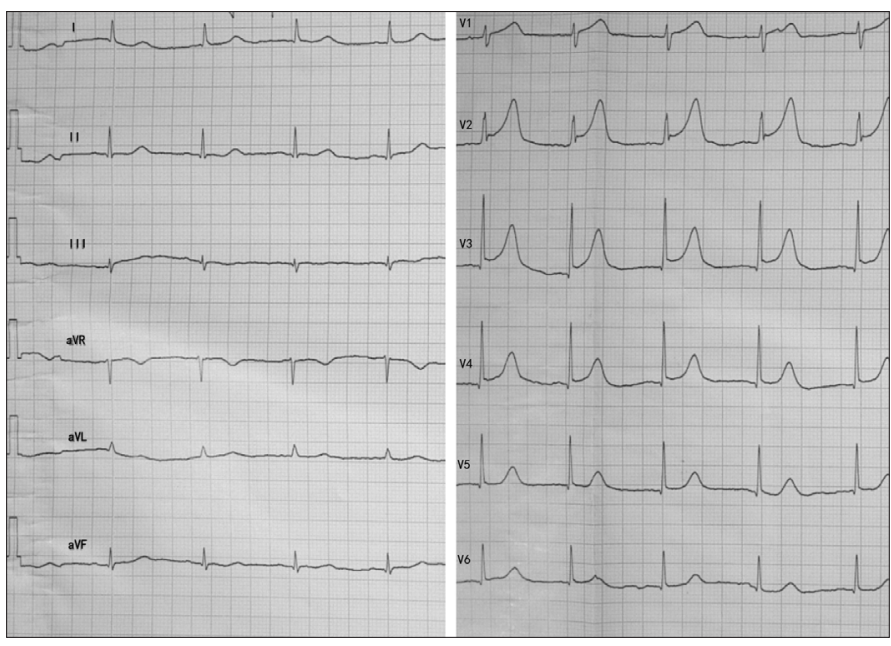

Figure 1. 12-lead electrocardiogram performed on presentation
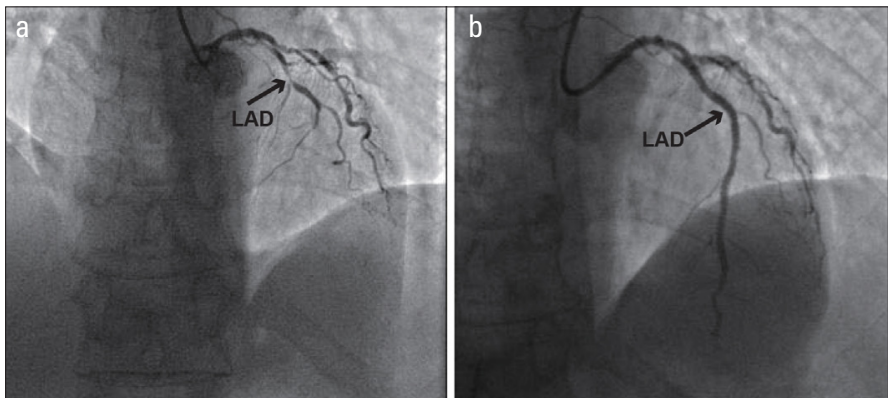

Figure 2. (a) Coronary angiography showed an acute occlusion of LAD. (b) Coronary angiography after PCI

$L A D$ - left anterior descending; $\mathrm{PCl}$ - percutaneous coronary intervention
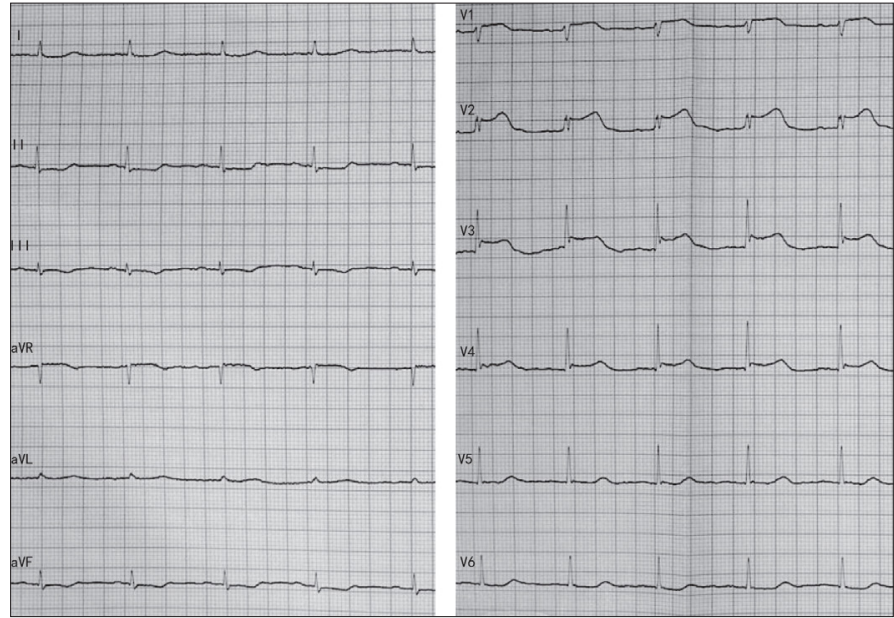

Figure 3. Electrocardiogram performed after percutaneous coronary intervention

\section{Discussion}

The transient prominent anterior QRS forces, as shown in Figure 1 , are the hallmark of LSFB. LSFB is a relatively common ECG manifestation, but very easily overlooked. The criteria for 


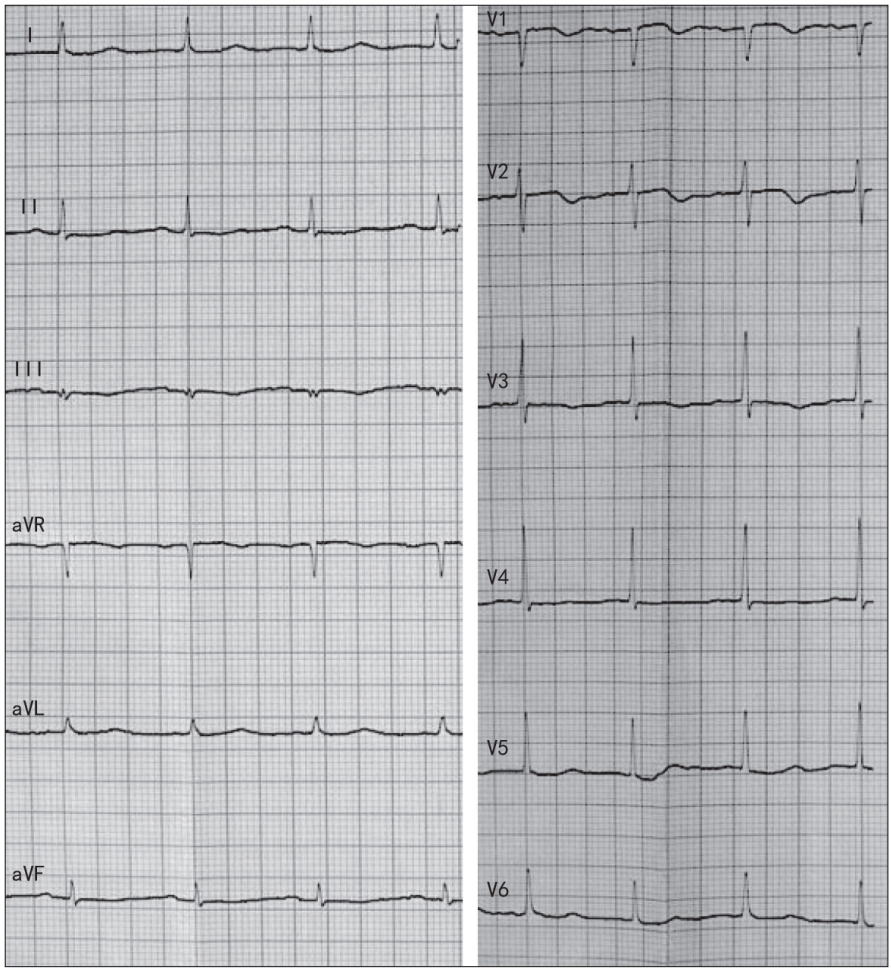

Figure 4. Electrocardiogram performed on the day after percutaneous coronary intervention

ECG diagnosis of LSFB are as follows-ORS duration $<120 \mathrm{~ms}$ in general, close to $100 \mathrm{~ms}$; more than $15 \mathrm{~mm}$ voltage $R$ waves in V2 and V3 or from V1; increasing for all intermediary precordial leads and decreasing from V5 to V6; " $R$ " wave jump may occur from V1 to V2 ("rS" in V1 for R in V2); absence of QRS axis shift in the frontal plane; and $T$ wave polarity negative in the right precordial leads most of the time (1). LSF was first described by Dr Sunao Tawara, just as LAF and LPF (2).

Although LAFB and LPFB are well known, LSFB is easily overlooked by physicians, especially in the case of ischemic heart disease. Although LAF and LPF may be irrigated by the LAD coronary artery and right coronary artery, LSF is irrigated exclusively by $L A D$, and therefore, $L A D$ occlusion can result in $L S F B$. LSFB exclusively affects precordial leads without modifying the ECG in the frontal plane, causing prominent anterior QRS forces.
Other possible etiologies of LSFB are chronic Chagas cardiomyopathy, nonobstructive hypertrophic cardiomyopathy, obstructive hypertrophic cardiomyopathy, aortic valve disease, diabetes mellitus, and Kearns-Sayre syndrome (3). The transitory nature of the electrocardiographic changes rules out any other possible cause.

\section{Conclusion}

We report a case of transient LSFB caused by the acute occlusion of the proximal LAD. Because transient LSFB may indicate critical obstructive lesions of the proximal $L A D$, but is easily overlooked, physicians should pay more attention to this ECG pattern.

Funding: This work was supported by the National Natural Science Foundation (No. 81800292), The Seed Plan Program of Beijing Friendship Hospital (YYZZ2018A01) and the Rising Star Program from Beijing Friendship Hospital (No.YYODKT2017-16).

Informed consent: Informed consent was obtained from the patient.

\section{References}

1. Sociedade Brasileira de Cardiologia. Guidelines of Sociedade Brasileira de Cardiologia about analysis and issuance of expert opinion in electrocardiographic (2009). Arq Bras Cardiol 2009; 93 (3 Suppl 2): 2-19. [Portuguese]

2. Silverman ME, Grove D, Upshaw CB Jr. Why does the heart beat? The discovery of the electrical system of the heart. Circulation 2006; 113: 2775-81. [Crossref]

3. Pérez-Riera AR, Barbosa-Barros R, Baranchuk A. Left septal fascicular block: Characterization, differential diagnosis and clinical significance. London, UK: Springer Publishing Company; 2016. [Crossref]

Address for Correspondence: Hongwei Li, MD,

Department of Cardiology, Cardiovascular Center, Beijing Friendship Hospital, Capital Medical University; Beijing-China

Phone: 861063138344

E-mail: Ihw1965@sina.com

CCopyright 2021 by Turkish Society of Cardiology Available online at www.anatoljcardiol.com DOI:10.5152/AnatolJCardiol.2021.93490 